\title{
A Systematic Literature Review on Technology-Enhanced Learning in Medical Education
}

\author{
May Honey Ohnn", Bareth Ravindran², Chan Zhi Weï ${ }^{3}$, Khin Maung Ohn", Ng Pey Luen ${ }^{5}$
}

\author{
${ }^{1}$ Emergency Department, Sandwell \& West \\ Birmingham NHS Trust, United Kingdom \\ 2 Surgical Department, \\ Hospital Tuanku Ja'afar Seremban, \\ Kuala Lumpur, Malaysia \\ ${ }^{3}$ Orthopaedics Department, \\ Hospital Sungai Buloh, Kuala Lumpur, Malaysia \\ ${ }^{4}$ Department of Surgery, Faculty of Medicine \\ and Health Sciences, Universiti Malaysia \\ Sabah, Kota Kinabalu, Sabah, Malaysia \\ ${ }^{5}$ Radiology Department, \\ Hospital Queen Elizabeth II, Sabah, Malaysia
}

\section{*Corresponding author's email: mayhoney.ohn@gmail.com}

Received: 14 July 2019

Accepted: 5 November 2019

Keywords:

blended learning, technology-

enhanced learning, medical

education, hybrid learning,

undergraduate medical students

\section{ABSTRACT}

This systematic review was aimed to evaluate the effectiveness of technology-enhanced learning (TEL) used in medical teaching for undergraduate medical students. The objectives are to (a) identify various teaching modalities which are used to enhance TEL; (b) evaluate whether TEL is more effective than traditional learning $(T L)$ in cognitive and affective learning domain outcome. The empirical studies were searched in the following databases: Google Scholar, MEDLINE, ERIC, ProQuest, Cochrane Library and Scopus. All papers published from 2008 to 2018 were included. From eligible studies, the study design, study field, study population, intervention methods, type of assessment and learning achievement were abstracted and summarized the information. The search results were independently reviewed by two authors. From a pool of 1384 articles, 43 eligible articles were identified, enrolling 7292 undergraduate medical students examining comparative study between TEL and traditional learning. The results showed that there was a high degree of heterogeneity seen amongst the included studies in terms of TEL modalities used. The majority of intervention studies favoured online resources, while the rest used various offline multimedia electronic devices, virtual simulations and blended modes. Overall findings showed promising data that TEL is better than TL with regards to knowledge gain and skill acquisition, as well as providing higher student satisfaction. In all, the findings present blended learning in a positive and promising light in time, particularly where systematic reviews on technology-enhanced learning in the field of the undergraduate medical programme have produced mixed result. 


\section{INTRODUCTION}

Traditionally, the instructional method in medical education has always been in the form of face-to-face teaching1, 2. With traditional learning ( $T L)$, the majority of the allocated time is devoted to information transfer from a content expert to a novice student. The extent to which students partake with the content is highly variable, although small-group teaching certainly opens the window for direct interaction between the learner and the teacher, permitting immediate feedback and clarification between the two. Nonetheless, large-group sessions such as lectures are often relatively impersonal ${ }^{3}$ and students may find this approach is not ideal for their learning ${ }^{4}$ and the status of webbased education in Saudi higher education are demonstrated. Three main challenges of applying blended learning in Saudi higher education are addressed. One major challenge to be considered in the implementation of blended learning in Saudi universities is the adaptation of this element in the traditional university culture. Finding the right design of blended learning is another challenge that is discussed in detail. Furthermore, the time issue is considered a crucial challenge facing blended learning faculty. Practical recommendations that would facilitate transition to a blended learning university environment are presented. It is hoped that this study will help to provide insight for the faculties and the decision-makers throughout higher education in Saudi Arabia. Although this investigation is specifically related to the implementation of blended learning in the universities of Saudi Arabia, we are confident that the assumptions and recommendations contained herein will be of great value to other populations facing similar challenges. Introduction The Saudi Ministry of Higher Education has encouraged the use of information tech-nology (IT. In addition, the learning process in the traditional setting also emphasises more on student learning "what" and not "how". The primary focus also tends to be more on completing the required subject matter quota with minimal to no student involvement in inquiry-based education and in solving problems, but rather in tasks set by the teacher. This leads to low motivation and the subject matter is "distant" to the students. Teachers hold an authoritarian role and tend to dictate the structure of the lesson and the division of time. The learning process takes place within a classroom and school in accordance with a designated timetable, resulting in students having no flexibility 5 .

Nevertheless, the past two decades have seen a revolutionizing transformation in the innovations of technology and devices, and the field of medical education must evolve accordingly to catch up ${ }^{6}$. Technologyenhanced learning (TEL) which is used synonymously with e-learning, does not have a unique definition. It is typically used to describe the utilization of information technology (IT) to educating and learning students. When TEL is combined systematically with faceto-face teaching to increase communication among students, instructors, and resources, it is regarded as blended learning $(\mathrm{BL})^{7}$. Blended learning, which allows flexibility and creativity, are nowadays popular teaching strategy in medical institutions. Although initially met with scepticism, there has been an increased acceptance of technology-enhanced blended learning in the field of medical education ${ }^{8}$, ${ }^{9}$ and it is ludicrous that any educational establishment, at any level from school to the higher education institution, would consider withdrawing technology from their curriculum $^{10,11}$. and it is ludicrous that any educational establishment, at any level from school to the higher education institution, would consider withdrawing technology from their curriculum.

The exact prevalence and beneficial outcome of the usage of TEL in medical institutions are yet determined but it was found that most institutions of higher educations in Malaysia have adequate infrastructure 
for e-learning, equipped with broadband internet access and some with wireless mobile computing capabilities ${ }^{12}$. Globally, various modalities of TEL resources are being introduced in medical education, with some success in their implementation ${ }^{13}-{ }^{15}$ and novice students often experience difficulty grasping the complex three-dimensional (3D. If medical education in Malaysia is to maintain a competitive edge, it needs to keep abreast of contemporarydevelopments. Hence, itishoped that this systematic review would highlight the impact of TEL on undergraduate medical education, which may justify its consolidation into the local medical curriculum. The aim and objectives of this systematic review are to (i) identify various teaching modalities which are used to enhance TEL among undergraduate medical students, (ii) establish whether TEL achieves better improvement in a cognitive learning outcome, (iii) establish whether TEL achieves better improvement in affective learning outcome. It was hypothesized that overall learning effectiveness would be higher with TEL than TL.

\section{MATERIALS AND METHODS}

\section{Search Strategy}

The systematic review was carried out using a comprehensive search strategy with selection criteria. Two authors (MHO and $\mathrm{KMO}$ ) independently searched the empirical studies in the following databases: Google Scholar, MEDLINE, ERIC, ProQuest, CINAHL, Embase, PsycINFO, Cochrane Library, Scopus. It was conducted in accordance with the Cochrane Collaboration guideline ${ }^{16}$ to answer the research objectives, risk of bias, appropriateness of outcome measures and generalizability of results. All papers published in English languages from 2008 to
2018 were included in this systematic review. Search criteria used MeSH terms which are refined using keywords of published articles. Search terms are connected using the Boolean Operators 'AND' and 'OR' to capture all relevant article suggestions. A total of 20 synonyms were used to identify key papers relevant to two concepts in the peer-reviewed scientific literature i.e. electronic learning and undergraduate medical education. These phrases are "undergraduate medical education" OR "Undergraduate Medical Students" OR "Clinical-year students" OR "Pre-clinical year students" AND "technologyenhanced learning" OR "e-learning" OR "webbased learning" OR "internet-based learning" OR "m-learning" OR "computer-assisted learning" OR "online learning" AND "Traditional teaching" OR "Face-to-face teaching" AND "learning effectiveness" OR "learning achievement" OR "cognitive learning outcome" $O R$ "knowledge gain" OR "skill acquisition" OR "Affective learning outcome" OR "satisfaction". Cross-references were checked from the selected papers not to miss the hidden papers. Those duplicated papers were excluded from the final analysis.

\section{Ethical Considerations}

The ethical consideration was not necessary as this review consisted of a secondary analysis of published articles.

\section{Study Eligibility}

Population, Intervention, Comparator, outcome and study design (PICOS) framework for systematic review which has been endorsed by the Cochrane Collaboration, is used in this study ${ }^{17}$. The study considered to include in this systematic review only if it fulfils all inclusion criteria and does not meet any of the exclusion criteria as outlined in Table 1. 
Borneo Journal of Medical Sciences 14 (1) January, 2020: $3-17$

Table 1 Criteria to assess the eligibility by using Population, Intervention, Comparison, Outcome and Study design (PICOS) framework to be included in this systematic review

\begin{tabular}{|l|l|l|}
\hline Parameter & Inclusion criteria & Exclusion criteria \\
\hline Participants & $\begin{array}{l}\text { All undergraduate medical students (pre-clinical year and/or } \\
\text { clinical-year students) }\end{array}$ & $\begin{array}{l}\text { Postgraduate medical students } \\
\text { Non-medical students } \\
\text { Nursing students }\end{array}$ \\
\hline Intervention & Any technology-enhanced learning & No technology involved \\
\hline Comparison & Face-to-face or traditional or didactic learning & No comparison or control group \\
\hline Outcomes & $\begin{array}{l}\text { Level 1 \& 2 Kirkpatrick evaluation model }{ }^{18} \text { were used to assess } \\
\text { the learning effectiveness or achievement in terms of cognitive } \\
\text { learning outcome (knowledge gain and/or skill acquisition) and/ } \\
\text { or affective learning outcome (learning satisfaction). }\end{array}$ & \\
\hline Study design & $\begin{array}{l}\text { Quantitative Comparative interventional study design } \\
\text { Qualitative study }\end{array}$ \\
\hline
\end{tabular}

\section{Validity, Reliability and Rigour}

This review was conducted in adherence to PRISMA standards of quality of reporting systematic review and meta-analyses ${ }^{19}$ (Figure 1).

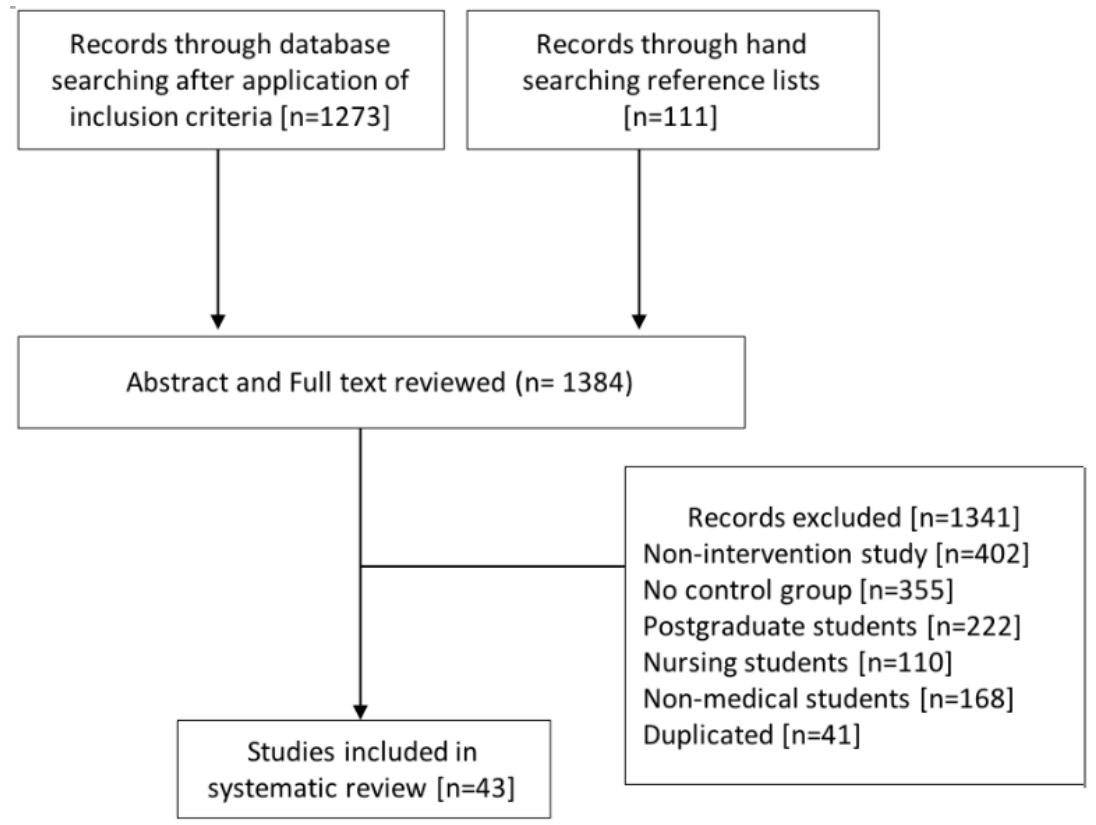

Figure 1 PRISMA flowchart of the systematic review

\section{Data Extraction and Analysis}

From eligible studies, the study subject, study population, instructional methods, type of assessment and learning achievement were abstracted and summarized the information. Two authors (CZW and BR) independently reviewed the search results and screened data using pre-defined extraction templates. Another reviewer (NPL) was involved where disagreements arose over the relevancy of the data. The results were downloaded to Mendeley where duplicate citations were removed. Data extraction and analysis were done using Microsoft Excel'. Descriptive statistics were described in terms of frequency. The systematic review was limited by the presence of selection and/or performance bias found in some of the selected studies. 


\section{RESULTS}

Table 2 shows detail information extracted from this review. There were 44 published studies included in the review, a vast majority of which (33 of them) were Randomized Controlled Trials (RCT), with the rest being 1 comparative study, 3 randomised cross over studies, 2 quasi-experimental studies, 2 cohort studies and 2 mixed methods paper. A total of 7,292 undergraduate students were involved in the systematic review. A high degree of heterogeneity is seen amongst the included studies in terms of TEL modalities used. Twenty-two out of the forty-three studies (51\%) utilized online resources, which included online lectures, online courses, e-learning portals, discussion forums and emails. Multimedia or electronic devices, including podcasts, digital game-based learning, CDROM, mobile devices and videos, were used by 13 studies (30\%). Eight studies of the fortythree (19\%) intervened with virtual models, such as augmented reality and virtual reality environment, as well as 3D models. As seen, a wide range of technology was used by the studies, all in different ways to improve and enhance medical student learning. Several individual technologies were mentioned specifically, such as augmented VR for anatomy and histology, Artificial Interface for Clinical Education, Virtual Microscopy, webcast and online 3D anatomy module. However, some studies do not describe their intervention in detail, making replication of these studies in other domains difficult. It is also noted that thirty-nine out of forty-three (91\%) studies were done in the developed countries (United States, Canada, Europe, United Kingdom, and Australia); while the remaining four (9\%) came from Asia (China and India), with none from South America, Africa, and the Middle East.

Table 2 Summary of the study design, population, intervention, assessment and outcomes of the included studies.

\begin{tabular}{|c|c|c|c|c|c|c|c|}
\hline Author (year) & Study design & Study population & $\begin{array}{l}\text { Geographic } \\
\text { location }\end{array}$ & $\begin{array}{l}\text { Numbers of } \\
\text { participants }\end{array}$ & $\begin{array}{l}\text { Study } \\
\text { intervention }\end{array}$ & $\begin{array}{l}\text { Study } \\
\text { assessment }\end{array}$ & Study outcome \\
\hline $\begin{array}{l}\text { Ackermann et al. } \\
(2010)^{20}\end{array}$ & RCT & $\begin{array}{l}\text { 3rd-year medical } \\
\text { students }\end{array}$ & $\begin{array}{l}\text { Germany } \\
\text { (Europe) }\end{array}$ & 19 & $\begin{array}{l}\text { Software CD } \\
\text { vs traditional } \\
\text { learning }\end{array}$ & Pre and post-test & $\begin{array}{l}\text { 1. X-ray } \\
\text { interpretation } \\
\text { skill: Results } \\
\text { favour TEL } \\
\text { over TL }\end{array}$ \\
\hline Allen et al. $(2016)^{13}$ & RCT & $\begin{array}{l}\text { Undergraduate } \\
\text { medical students }\end{array}$ & Canada & 47 & $\begin{array}{l}\text { Online 3D } \\
\text { neuroanatomy } \\
\text { interactive } \\
\text { module vs } \\
\text { traditional } \\
\text { laboratory module }\end{array}$ & Pre and post-test & $\begin{array}{l}\text { 1. Knowledge: } \\
\text { Results favour } \\
\text { TEL over TL } \\
\text { 2. Satisfaction: } \\
\text { Results favour } \\
\text { TEL over TL }\end{array}$ \\
\hline Amesse $(2008)^{21}$ & RCT & $\begin{array}{l}\text { 3rd-year medical } \\
\text { students }\end{array}$ & USA & 36 & $\begin{array}{l}\text { Computer- } \\
\text { based learning } \\
\text { tutorial session } \\
\text { vs traditional } \\
\text { learning }\end{array}$ & Post-test & $\begin{array}{l}\text { 1.Knowledge: } \\
\text { Results favour } \\
\text { TEL over TL }\end{array}$ \\
\hline $\begin{array}{l}\text { Armstrong et al. } \\
(2009)^{22}\end{array}$ & RCT & $\begin{array}{l}\text { 4th-year medical } \\
\text { students }\end{array}$ & United Kingdom & 21 & $\begin{array}{l}\text { Interactive } \\
\text { slide show } \\
\text { vs traditional } \\
\text { learning }\end{array}$ & Post-test & $\begin{array}{l}\text { 1. Knowledge: } \\
\text { No significant } \\
\text { difference } \\
\text { between TEL } \\
\text { and TL }\end{array}$ \\
\hline
\end{tabular}


Borneo Journal of Medical Sciences 14 (1) January, 2020: 3-17

\begin{tabular}{|c|c|c|c|c|c|c|c|}
\hline Davidson $(2011)^{23}$ & Cohort & $\begin{array}{l}\text { 1st-year medical } \\
\text { students }\end{array}$ & Canada & 300 & $\begin{array}{l}\text { Blended online } \\
\text { team-based } \\
\text { learning vs } \\
\text { didactic learning }\end{array}$ & $\begin{array}{l}\text { 1. Course } \\
\text { evaluation survey } \\
\text { with Likert scale } \\
\text { 2.Examination } \\
\text { results }\end{array}$ & $\begin{array}{l}\text { 1. Knowledge: } \\
\text { Results favour } \\
\text { TEL over TL } \\
\text { 2. Satisfaction: } \\
\text { Results favour } \\
\text { TEL over TL }\end{array}$ \\
\hline $\begin{array}{l}\text { Ferrer-Torregrosa et al. } \\
(2015)^{24}\end{array}$ & RCT & $\begin{array}{l}\text { 1st-year medical } \\
\text { students }\end{array}$ & Spain (Europe) & 211 & $\begin{array}{l}\text { Augmented } \\
\text { reality book vs } \\
\text { standard sessions } \\
\text { with lectures, } \\
\text { slides, and video } \\
\text { recordings of } \\
\text { cadaveric material }\end{array}$ & 1. Post-test & $\begin{array}{l}\text { Knowledge: } \\
\text { Results favour } \\
\text { TEL over TL }\end{array}$ \\
\hline Green et al. $(2011)^{25}$ & RCT & $\begin{array}{l}\text { 2nd-year medical } \\
\text { students }\end{array}$ & USA & 121 & $\begin{array}{l}\text { Interactive } \\
\text { computer- } \\
\text { based program } \\
\text { vs. traditional } \\
\text { learning }\end{array}$ & $\begin{array}{l}\text { 1. Post-test } \\
\text { 2. Questionnaire }\end{array}$ & $\begin{array}{l}\text { 1. Knowledge: } \\
\text { Results favour } \\
\text { TEL over TL }\end{array}$ \\
\hline Gunn et al. $(2017)^{26}$ & RCT & $\begin{array}{l}\text { 1st-year medical } \\
\text { imaging students }\end{array}$ & Australia & 33 & $\begin{array}{l}\text { Virtual reality } \\
\text { radiology } \\
\text { simulation } \\
\text { software vs } \\
\text { traditional } \\
\text { radiology } \\
\text { laboratory session }\end{array}$ & Post-test & $\begin{array}{l}\text { 1. Knowledge: } \\
\text { Results favour } \\
\text { TEL over TL } \\
\text { 2. Skill: Results } \\
\text { favour TEL } \\
\text { over TL }\end{array}$ \\
\hline $\begin{array}{l}\text { Haeur et al. }(2009)^{27} \mathrm{p} \\
=.01\end{array}$ & RCT & $\begin{array}{l}\text { 3rd-year medical } \\
\text { students }\end{array}$ & USA & 303 & $\begin{array}{l}\text { Web-based } \\
\text { learning vs control } \\
\text { group }\end{array}$ & $\begin{array}{l}\text { 1. Checklist } \\
\text { 2.8-item } \\
\text { satisfaction } \\
\text { survey with } \\
\text { responses on a } \\
\text { Likert type scale }\end{array}$ & $\begin{array}{l}\text { 1. Skills: Results } \\
\text { favour TEL } \\
\text { over TL } \\
\text { 2. Satisfaction: } \\
\text { Results favour } \\
\text { TEL over TL }\end{array}$ \\
\hline llic et al. $(2015)^{28}$ & Mixed method & $\begin{array}{l}\text { 1st-year medical } \\
\text { students }\end{array}$ & Australia & 147 & $\begin{array}{l}\text { Blended learning } \\
\text { (online plus } \\
\text { classroom } \\
\text { plus mobile) } \\
\text { vs traditional } \\
\text { learning }\end{array}$ & $\begin{array}{l}\text { 1. Berlin } \\
\text { questionnaire- } \\
\text { 2. Assessing } \\
\text { Competency in } \\
\text { EBM (ACE) tool - } \\
15 \mathrm{MCQ} \\
\text { 3. Evidence- } \\
\text { based Practice } \\
\text { Question (EBPQ) } \\
\text {, focused group } \\
\text { discussion }\end{array}$ & $\begin{array}{l}\text { 1. Knowledge: } \\
\text { Results favour } \\
\text { TEL over TL } \\
\text { 2. Satisfaction: } \\
\text { Results favour } \\
\text { TEL over TL }\end{array}$ \\
\hline Ingrassia et al. (2014) & RCT & $\begin{array}{l}\text { 2nd-year medical } \\
\text { students }\end{array}$ & Italy & 524 & $\begin{array}{l}\text { Blended learning } \\
\text { (e-learning } \\
\text { plus Problem- } \\
\text { based learning) } \\
\text { vs. traditional } \\
\text { learning }\end{array}$ & $\begin{array}{l}\text { 1. Pre-test } \\
\text { and post-test, } \\
\text { simulation } \\
\text { exercises } \\
\text { 2. Likert scale } \\
\text { to assess } \\
\text { satisfaction }\end{array}$ & $\begin{array}{l}\text { 1. Knowledge: } \\
\text { Results favour } \\
\text { TEL over TL } \\
\text { 2. Satisfaction: } \\
\text { Results favour } \\
\text { TEL over TL }\end{array}$ \\
\hline $\begin{array}{l}\text { Javadian \& Shobeiri } \\
(2016)^{30}\end{array}$ & RCT & $\begin{array}{l}\text { 3rd-year medical } \\
\text { students }\end{array}$ & USA & 745 & $\begin{array}{l}\text { Internet-based } \\
\text { (IB) education vs. } \\
\text { dissection-based } \\
\text { (DB) education on } \\
\text { pelvic anatomy }\end{array}$ & Post-test & $\begin{array}{l}\text { Knowledge: } \\
\text { Results favour } \\
\text { TEL over TL }\end{array}$ \\
\hline
\end{tabular}




\begin{tabular}{|c|c|c|c|c|c|c|c|}
\hline Jenkins et al. $(2008)^{31}$ & RCT & $\begin{array}{l}\text { 2nd-year medical } \\
\text { students }\end{array}$ & USA & 73 & $\begin{array}{l}\text { Computer- } \\
\text { assisted } \\
\text { instruction tutorial } \\
\text { vs. traditional } \\
\text { lecture and group } \\
\text { work }\end{array}$ & Post-test & $\begin{array}{l}\text { 1. Knowledge: } \\
\text { No significant } \\
\text { difference } \\
\text { between TEL } \\
\text { and TL }\end{array}$ \\
\hline $\begin{array}{l}\text { Kandasamy \& Fung } \\
(2009)^{32}\end{array}$ & RCT & $\begin{array}{l}\text { 2nd-year medical } \\
\text { students }\end{array}$ & Canada & 55 & $\begin{array}{l}\text { Online computer- } \\
\text { assisted module } \\
\text { vs. review article }\end{array}$ & $\begin{array}{l}\text { 1. Post-test } \\
\text { 2. Questionnaire }\end{array}$ & $\begin{array}{l}\text { 1. Knowledge: } \\
\text { Results favour } \\
\text { TEL over TL } \\
\text { 2. Satisfaction: } \\
\text { Results favour } \\
\text { TEL over TL }\end{array}$ \\
\hline Kanthan $(2011)^{33}$ & RCT & $\begin{array}{l}\text { 1st and 2nd-year } \\
\text { medical students }\end{array}$ & Canada & 114 & $\begin{array}{l}\text { Digital games vs } \\
\text { no games }\end{array}$ & $\begin{array}{l}\text { 1. Pre-test and } \\
\text { post-test } \\
\text { 2. Satisfaction } \\
\text { survey } \\
\text { questionnaire }\end{array}$ & $\begin{array}{l}\text { 1. Knowledge: } \\
\text { Results favour } \\
\text { TEL over TL } \\
\text { 2. Satisfaction: } \\
\text { Results favour } \\
\text { TEL over TL } \\
\text { 3. Engagement: } \\
\text { Results favour } \\
\text { TEL over TL }\end{array}$ \\
\hline $\begin{array}{l}\text { Kerfoot \& Brotschi } \\
(2009)^{34}\end{array}$ & RCT & $\begin{array}{l}\text { 3rd, 4th and } \\
\text { 5th-year medical } \\
\text { students }\end{array}$ & USA & 115 & $\begin{array}{l}\text { Online spaced } \\
\text { education vs. } \\
\text { traditional } \\
\text { classroom }\end{array}$ & $\begin{array}{l}\text { Pre-test and } \\
\text { post-test }\end{array}$ & $\begin{array}{l}\text { Knowledge: } \\
\text { Results favour } \\
\text { TEL over TL }\end{array}$ \\
\hline Kleinert et al. $(2015)^{35}$ & RCT & $\begin{array}{l}\text { 3rd-year medical } \\
\text { students }\end{array}$ & Germany & 62 & $\begin{array}{l}\text { Virtual simulator } \\
\text { "ALICE" (Artificial } \\
\text { Interface for } \\
\text { Clinical Education) } \\
\text { vs. control group }\end{array}$ & $\begin{array}{l}\text { 1. Pre-test and } \\
\text { post-test } \\
\text { 2.6-point Likert } \\
\text { scale to assess } \\
\text { satisfaction }\end{array}$ & $\begin{array}{l}\text { 1. Knowledge: } \\
\text { Results favour } \\
\text { TEL over TL } \\
\text { 2. Satisfaction: } \\
\text { Results favour } \\
\text { TEL over TL }\end{array}$ \\
\hline Kong et al. $(2009)^{36}$ & RCT & $\begin{array}{l}\text { 5th-year medical } \\
\text { students }\end{array}$ & China & 90 & $\begin{array}{l}\text { PBL teaching } \\
\text { with digital } \\
\text { format material } \\
\text { vs. traditional } \\
\text { learning }\end{array}$ & $\begin{array}{l}\text { 1. Theoretical } \\
\text { and case analysis } \\
\text { examinations } \\
\text { 2. Evaluation of } \\
\text { students' practice } \\
\text { 3. Questionnaire }\end{array}$ & $\begin{array}{l}\text { 1. Knowledge: } \\
\text { Results favour } \\
\text { TEL over TL } \\
\text { 2. Skills: Results } \\
\text { favour TEL } \\
\text { over TL }\end{array}$ \\
\hline Langdorf et al. $(2018)^{37}$ & $\begin{array}{l}\text { Comparative } \\
\text { study }\end{array}$ & $\begin{array}{l}\text { 4th-year medical } \\
\text { students }\end{array}$ & USA & 468 & $\begin{array}{l}\text { Team-based } \\
\text { simulation } \\
\text { learning/Flipped } \\
\text { classroom vs. } \\
\text { didactic lecture } \\
\text { for ACLS }\end{array}$ & $\begin{array}{l}1.50 \text { multiple- } \\
\text { choice (MC) } \\
\text { format questions } \\
2.20 \text { rhythm- } \\
\text { matching } \\
\text { questions } \\
3.7 \text { fill-in } \\
\text { management of } \\
\text { simulated cases. }\end{array}$ & $\begin{array}{l}\text { 1. Knowledge: } \\
\text { Results favour } \\
\text { TEL over TL }\end{array}$ \\
\hline Lewis et al. $(2011)^{38}$ & RCT & $\begin{array}{l}\text { 2nd-year medical } \\
\text { students }\end{array}$ & Canada & 39 & $\begin{array}{l}\text { Access-to- } \\
\text { localization tool } \\
\text { exploring cranial } \\
\text { nerve lesions vs. } \\
\text { control group }\end{array}$ & $\begin{array}{l}\text { 1. Final } \\
\text { examination } \\
\text { scores } \\
\text { 2.Questionnaire }\end{array}$ & $\begin{array}{l}\text { 1. Knowledge: } \\
\text { Results favour } \\
\text { TEL over TL } \\
\text { 2. Satisfaction: } \\
\text { Results favour } \\
\text { TEL over TL }\end{array}$ \\
\hline
\end{tabular}


Borneo Journal of Medical Sciences 14 (1) January, 2020: 3-17

\begin{tabular}{|c|c|c|c|c|c|c|c|}
\hline Miller $(2016)^{39}$ & RCT & $\begin{array}{l}\text { 1st-year medical } \\
\text { students }\end{array}$ & USA & 265 & $\begin{array}{l}\text { Comparing } \\
\text { dissection of } \\
\text { donor cadavers } \\
\text { (qC), manipulation } \\
\text { of digitized 3D } \\
\text { holographic } \\
\text { renderings (qH) } \\
\text { and examination } \\
\text { of plastinated } \\
\text { specimens (qP) }\end{array}$ & $\begin{array}{l}\text { Class } \\
\text { examination } \\
\text { results }\end{array}$ & $\begin{array}{l}\text { Knowledge: } \\
\text { Results favour } \\
\text { TEL over TL }\end{array}$ \\
\hline Mione et al. $(2013)^{40}$ & $\begin{array}{l}\text { Randomised cross } \\
\text { over design }\end{array}$ & $\begin{array}{l}\text { Undergraduate } \\
\text { medical students }\end{array}$ & Belgium & 199 & $\begin{array}{l}\text { Virtual microscopy } \\
\text { (VM) vs. Light } \\
\text { microscopy (LM) }\end{array}$ & $\begin{array}{l}\text { Pre-test and } \\
\text { post-test with } \\
\text { crossover }\end{array}$ & $\begin{array}{l}\text { Knowledge: } \\
\text { No significant } \\
\text { difference } \\
\text { between TEL } \\
\text { and TL }\end{array}$ \\
\hline $\begin{array}{l}\text { Montassiera et al. } \\
(2016)^{41}\end{array}$ & RCT & $\begin{array}{l}\text { 5th-year medical } \\
\text { students }\end{array}$ & France & 89 & $\begin{array}{l}\text { ECG online } \\
\text { module vs. } \\
\text { traditional } \\
\text { learning }\end{array}$ & $\begin{array}{l}\text { ECG } \\
\text { interpretation } \\
\text { score }\end{array}$ & $\begin{array}{l}\text { 1. Knowledge: } \\
\text { No significant } \\
\text { difference } \\
\text { between TEL } \\
\text { and TL } \\
\text { 2. Satisfaction: } \\
\text { Results favour TL } \\
\text { over TEL }\end{array}$ \\
\hline $\begin{array}{l}\text { Ochoa \& Wludyka } \\
(2008)^{42}\end{array}$ & RCT & $\begin{array}{l}\text { 3rd-year medical } \\
\text { students }\end{array}$ & USA & 38 & $\begin{array}{l}\text { Web-based } \\
\text { interactive } \\
\text { programme } \\
\text { vs. traditional } \\
\text { learning }\end{array}$ & $\begin{array}{l}\text { 1. MCQ } \\
\text { 2. Likert scale }\end{array}$ & $\begin{array}{l}\text { 1. Knowledge: } \\
\text { Results favour } \\
\text { TEL over TL } \\
\text { 2. Satisfaction: } \\
\text { No significant } \\
\text { difference } \\
\text { between TEL } \\
\text { over TL }\end{array}$ \\
\hline $\begin{array}{l}\text { Palmer \& Devitt } \\
(2008)^{43}\end{array}$ & RCT & $\begin{array}{l}\text { 4th-year medical } \\
\text { students }\end{array}$ & Australia & 130 & $\begin{array}{l}\text { Interactive } \\
\text { computer-based } \\
\text { format with } \\
\text { detailed feedback } \\
\text { vs. standard } \\
\text { lecture material } \\
\text { vs. both }\end{array}$ & $\begin{array}{l}\text { 1. Post-test } \\
\text { 2.Questionnaire }\end{array}$ & $\begin{array}{l}\text { 1. Knowledge: } \\
\text { No significant } \\
\text { difference } \\
\text { between TEL } \\
\text { and TL }\end{array}$ \\
\hline Pickering $(2017)^{14}$ & RCT & $\begin{array}{l}\text { 2nd-year medical } \\
\text { students }\end{array}$ & United Kingdom & 49 & $\begin{array}{l}\text { Anatomy drawing } \\
\text { screencast vs. } \\
\text { textbooks }\end{array}$ & $\begin{array}{l}\text { Pre-test and } \\
\text { post-test }\end{array}$ & $\begin{array}{l}\text { Knowledge: } \\
\text { Results favour } \\
\text { TEL over TL }\end{array}$ \\
\hline Raupach et al. (2009) ${ }^{44}$ & RCT & $\begin{array}{l}\text { 4th-year medical } \\
\text { students }\end{array}$ & Germany & 148 & $\begin{array}{l}\text { Web-based } \\
\text { collaborative } \\
\text { teaching module } \\
\text { vs. traditional } \\
\text { learning }\end{array}$ & $\begin{array}{l}\text { 1. Pre-test and } \\
\text { post-test } \\
\text { 2.Questionnaire }\end{array}$ & $\begin{array}{l}\text { 1. Knowledge: } \\
\text { Results favour } \\
\text { TEL over TL }\end{array}$ \\
\hline Raupach et al. $(2010)^{45}$ & RCT & $\begin{array}{l}\text { 4th-year medical } \\
\text { students }\end{array}$ & Germany & 74 & $\begin{array}{l}\text { Web-based } \\
\text { problem-based } \\
\text { learning group vs. } \\
\text { traditional group }\end{array}$ & $\begin{array}{l}\text { 1. Post-test } \\
\text { 2.Questionnaire }\end{array}$ & $\begin{array}{l}\text { 1. Knowledge: } \\
\text { No significant } \\
\text { difference } \\
\text { between TEL } \\
\text { and TL }\end{array}$ \\
\hline
\end{tabular}


A Systematic Literature Review on Technology-Enhanced Learning in Medical Education

\begin{tabular}{|c|c|c|c|c|c|c|c|}
\hline Ricks et al. $(2008)^{46}$ & RCT & $\begin{array}{l}\text { 3rd and 4th-year } \\
\text { medical students }\end{array}$ & Canada & 23 & $\begin{array}{l}\text { E-learning (web- } \\
\text { based tutorials) } \\
\text { vs. traditional } \\
\text { learning }\end{array}$ & Post-test & $\begin{array}{l}\text { 1. Knowledge: } \\
\text { Results favour } \\
\text { TEL over TL }\end{array}$ \\
\hline Saltarelli et al. (2014) & $\begin{array}{l}\text { Quasi- } \\
\text { experimental trail }\end{array}$ & $\begin{array}{l}\text { 1st and 4th-year } \\
\text { medical students }\end{array}$ & USA & 165 & $\begin{array}{l}\text { Model-based } \\
\text { multimedia } \\
\text { simulation } \\
\text { learning system } \\
\text { vs. traditional } \\
\text { undergraduate } \\
\text { human cadaver } \\
\text { laboratory }\end{array}$ & Post-test & $\begin{array}{l}\text { Knowledge: } \\
\text { Results favour TL } \\
\text { over TEL }\end{array}$ \\
\hline Schreiber et al. $(2010)^{48}$ & $\begin{array}{l}\text { Randomized cross } \\
\text { over study }\end{array}$ & $\begin{array}{l}\text { Undergraduate } \\
\text { medical students }\end{array}$ & United Kingdom & 100 & $\begin{array}{l}\text { Video podcast vs. } \\
\text { live lectures }\end{array}$ & $\begin{array}{l}\text { 1. MCQ } \\
\text { 2.Questionnaires } \\
\text { to assess } \\
\text { satisfaction }\end{array}$ & $\begin{array}{l}\text { 1. Knowledge: } \\
\text { No significant } \\
\text { difference } \\
\text { between TEL } \\
\text { and TL } \\
\text { 2. Satisfaction: } \\
\text { Results favour TL } \\
\text { over TEL }\end{array}$ \\
\hline Smits et al. $(2012)^{49}$ & RCT & $\begin{array}{l}\text { 2nd-year medical } \\
\text { students }\end{array}$ & Netherlands & 141 & $\begin{array}{l}\text { E-learning vs. } \\
\text { traditional } \\
\text { learning }\end{array}$ & $\begin{array}{l}\text { 1. Post-test } \\
\text { 2.5-point Likert } \\
\text { scale }\end{array}$ & $\begin{array}{l}\text { 1. Knowledge: } \\
\text { No significant } \\
\text { difference } \\
\text { between TEL } \\
\text { and TL }\end{array}$ \\
\hline Stolz et al. $(2012)^{50}$ & RCT & $\begin{array}{l}\text { 3rd-year medical } \\
\text { students }\end{array}$ & Switzerland & 129 & $\begin{array}{l}\text { E-learning vs. } \\
\text { traditional } \\
\text { learning }\end{array}$ & $\begin{array}{l}\text { 1. Post-test } \\
\text { 2. 12-item OSCE }\end{array}$ & $\begin{array}{l}\text { 1. Knowledge: } \\
\text { No significant } \\
\text { difference } \\
\text { between TEL } \\
\text { and TL } \\
\text { 2. Skills: Results } \\
\text { favour TL over } \\
\text { TEL }\end{array}$ \\
\hline $\begin{array}{l}\text { Subramanian et al. } \\
(2012)^{51}\end{array}$ & RCT & $\begin{array}{l}\text { 3rd-year medical } \\
\text { students }\end{array}$ & USA & 30 & $\begin{array}{l}\text { E-learning vs. } \\
\text { traditional } \\
\text { learning }\end{array}$ & $\begin{array}{l}\text { 1. Post-test } \\
\text { 2. Long term } \\
\text { post-test }\end{array}$ & $\begin{array}{l}\text { 1. Knowledge: } \\
\text { Results favour } \\
\text { TEL over TL }\end{array}$ \\
\hline Stirling \& Birt 2014) & $\begin{array}{l}\text { Randomized cross } \\
\text { over study }\end{array}$ & $\begin{array}{l}\text { 1st-year medical } \\
\text { students }\end{array}$ & Australia & 71 & $\begin{array}{l}\text { Enriched } \\
\text { multimedia eBook } \\
\text { vs. traditional } \\
\text { anatomy practical } \\
\text { session }\end{array}$ & Pre and post-test & $\begin{array}{l}\text { 1. Knowledge: } \\
\text { Not significant } \\
\text { different } \\
\text { between TEL } \\
\text { and TL } \\
\text { 2. Satisfaction: } \\
\text { Results favour TL } \\
\text { over TEL }\end{array}$ \\
\hline Tian et al. $(2014)^{53}$ & RCT & $\begin{array}{l}\text { 2nd-year medical } \\
\text { students }\end{array}$ & China & 229 & $\begin{array}{l}\text { Virtual microscopy } \\
\text { (VM) vs. Light } \\
\text { microscopy (LM) }\end{array}$ & $\begin{array}{l}\text { 1. Post-test } \\
\text { 2.Questionnaires } \\
\text { to assess } \\
\text { students' } \\
\text { teaching } \\
\text { preference and } \\
\text { satisfaction }\end{array}$ & $\begin{array}{l}\text { 1. Knowledge: } \\
\text { Results favour } \\
\text { TEL over TL } \\
\text { 2. Satisfaction: } \\
\text { Results favour } \\
\text { TEL over TL }\end{array}$ \\
\hline
\end{tabular}


Borneo Journal of Medical Sciences 14 (1) January, 2020: 3-17

\begin{tabular}{|c|c|c|c|c|c|c|c|}
\hline $\begin{array}{l}\text { Thompson \& Laurie } \\
(2017)^{54}\end{array}$ & Cohort study & $\begin{array}{l}\text { Undergraduate } \\
\text { medical students }\end{array}$ & USA & 1171 & $\begin{array}{l}\text { Virtual histology } \\
\text { module with } \\
\text { multiple } \\
\text { audiovisual } \\
\text { modalities and a } \\
\text { virtual microscope } \\
\text { platform vs. } \\
\text { traditional } \\
\text { laboratory } \\
\text { sessions }\end{array}$ & $\begin{array}{l}\text { 1. In-house } \\
\text { examination } \\
\text { results } \\
\text { 2. Evaluation } \\
\text { of trends in } \\
\text { performance on } \\
\text { the histology } \\
\text { and cell biology } \\
\text { portion of } \\
\text { the United } \\
\text { States Medical } \\
\text { Licensing } \\
\text { Examination } \\
\text { (USMLE) Step } 1 \\
\text { Examination } \\
\text { 3.Questionnaire } \\
\text { for student } \\
\text { feedback }\end{array}$ & $\begin{array}{l}\text { 1. Knowledge: } \\
\text { Results favour } \\
\text { TEL over TL } \\
\text { 2. Satisfaction: } \\
\text { Results favour } \\
\text { TEL over TL }\end{array}$ \\
\hline Truncali et al. $(2011)^{55}$ & RCT & $\begin{array}{l}\text { 1st-year medical } \\
\text { students }\end{array}$ & USA & 94 & $\begin{array}{l}\text { Interactive } \\
\text { multimedia slides } \\
\text { with external } \\
\text { resources and } \\
\text { videos vs. control } \\
\text { group }\end{array}$ & $\begin{array}{l}\text { 1. Post-test } \\
\text { 2. OSCE } \\
\text { 3. Questionnaire }\end{array}$ & $\begin{array}{l}\text { 1. Knowledge: } \\
\text { Results favour } \\
\text { TEL over TL } \\
\text { 2. Skills: Results } \\
\text { favour TEL } \\
\text { over TL } \\
\text { 3. Attitude: } \\
\text { Difference not } \\
\text { tested }\end{array}$ \\
\hline Webb \& Choi $(2014)^{56}$ & $\begin{array}{l}\text { Mixed-method } \\
\text { study }\end{array}$ & $\begin{array}{l}\text { 1st-year medical } \\
\text { student }\end{array}$ & United Kingdom & 165 & $\begin{array}{l}\text { Interactive } \\
\text { radiological } \\
\text { anatomy } \\
\text { e-learning module } \\
\text { vs. traditional } \\
\text { learning }\end{array}$ & $\begin{array}{l}\text { 1. Pre-test and } \\
\text { post-test } \\
\text { 2. Summative } \\
\text { course } \\
\text { assessment } \\
\text { by Integrated } \\
\text { Anatomy } \\
\text { Practical Paper } \\
\text { (IAPP) } \\
\text { 3. Questionnaire } \\
\text { to assess } \\
\text { satisfaction }\end{array}$ & $\begin{array}{l}\text { 1. Knowledge: } \\
\text { Results favour } \\
\text { TEL over TL } \\
\text { 2. Satisfaction: } \\
\text { Results favour } \\
\text { TEL over TL }\end{array}$ \\
\hline Vaccani et al. $(2016)^{15}$ & $\begin{array}{l}\text { Quasi- } \\
\text { experimental trial }\end{array}$ & $\begin{array}{l}\text { 3rd-year medical } \\
\text { students }\end{array}$ & Canada & 148 & $\begin{array}{l}\text { Webcast vs. } \\
\text { traditional live } \\
\text { lectures }\end{array}$ & $\begin{array}{l}\text { 1.Post-test } \\
\text { 2.Questionnaire } \\
\text { on students' } \\
\text { responses }\end{array}$ & $\begin{array}{l}\text { 1. Knowledge: } \\
\text { No significant } \\
\text { difference } \\
\text { between the two } \\
\text { groups } \\
\text { 2. Skills: Results } \\
\text { favour TEL } \\
\text { over TL } \\
\text { 3. Satisfaction: } \\
\text { Mixed results }\end{array}$ \\
\hline Vyas et al. $(2010)^{57}$ & RCT & $\begin{array}{l}\text { 4th-year medical } \\
\text { students }\end{array}$ & India & 52 & $\begin{array}{l}\text { Web-based } \\
\text { workshop vs. } \\
\text { control group }\end{array}$ & $\begin{array}{l}\text { 1. Clinical } \\
\text { reasoning } \\
\text { problems (CRP) } \\
\text { score } \\
\text { 2. Questionnaire }\end{array}$ & $\begin{array}{l}\text { 1. Knowledge: } \\
\text { Results favour } \\
\text { TEL over TL } \\
\text { 2. Satisfaction: } \\
\text { Results favour } \\
\text { TEL over TL }\end{array}$ \\
\hline
\end{tabular}




\begin{tabular}{|c|c|c|c|c|c|c|c|}
\hline Yeung et al. $(2012)^{58}$ & RCT & $\begin{array}{l}\text { 2nd-year medical } \\
\text { students }\end{array}$ & United Kingdom & 78 & $\begin{array}{l}\text { Online module vs. } \\
\text { control group }\end{array}$ & $\begin{array}{l}\text { 1. Post-test } \\
\text { 2. Subjective } \\
\text { questionnaire } \\
\text { using a 5-point } \\
\text { Likert scale }\end{array}$ & $\begin{array}{l}\text { 1. Knowledge: } \\
\text { No significant } \\
\text { difference } \\
\text { between TEL } \\
\text { and TL } \\
\text { 2. Satisfaction: } \\
\text { No significant } \\
\text { difference } \\
\text { between TEL } \\
\text { and TL }\end{array}$ \\
\hline Zeng Ru et al. $(2017)^{59}$ & RCT & Medical students & China & 181 & $\begin{array}{l}\text { ECG online } \\
\text { module vs. } \\
\text { traditional } \\
\text { learning }\end{array}$ & $\begin{array}{l}\text { ECG } \\
\text { interpretation } \\
\text { score }\end{array}$ & $\begin{array}{l}\text { 1. Knowledge: } \\
\text { Results favour } \\
\text { TEL over TL } \\
\text { 2. Satisfaction: } \\
\text { Results favour } \\
\text { TEL over TL }\end{array}$ \\
\hline
\end{tabular}

RCT: randomized controlled trial; TEL: technology-enhanced learning; TL: traditional learning; vs: versus; MCQ: multiple choice question; OSCE: objective structured clinical examination; ECG: electrocardiogram

Forty-two (97\%) selected papers have studied knowledge as a learning outcome. Thirty of the forty-two studies (71\%) found that usage of technology-enhanced blended learning has resulted in significantly improved knowledge among test subjects. On the other hand, 12 studies (28\%) found that there is no significant difference while only 1 study (2\%) favoured traditional learning over TEL. Notably, all three studies which used a blended learning method combining online learning and face-to-face teaching showed $100 \%$ favourable on knowledge improvement over TL. In addition, a total of 7 papers have studied skills as an outcome where 6 studies (86\%) have found that use of TEL resulted in significantly improved skills whereas a single study favours traditional learning. Next, a total of 20 studies have taken learner's satisfaction into consideration as an outcome. In 15 (75\%), the results favoured TEL over traditional learning whereas 3 papers $(15 \%)$ found learners to be more satisfied with traditional learning while another 2 (10\%) found no significant difference or mixed results.

\section{DISCUSSION}

Overall findings of the included studies show promising data that TEL is better than TL with regards to knowledge gain and skill acquisition, as well as providing higher student satisfaction. A review published in 2012 reported that blended learning in the clinical education of healthcare students shows some measure of improvement in students' competencies, and further suggests that the highly contextual, complex needs of a competent healthcare graduate can potentially be addressed by blended learning ${ }^{60}$. On the other hand, a 2015 WHO review have suggested that in terms of knowledge and skill gain, TEL is "no better and no worse" than traditional learning for undergraduate healthcare profession learning ${ }^{61}$. However, our review cannot be entirely compared to the other presently available reviews, as the reviews lack focus solely on undergraduate medical students, including both clinical and pre-clinical groups. They are either generalized for all fields of healthcare profession students, or for niche groups within medical education. 
Amongst the various modalities studied, online resources are favoured and often utilized in medical education, because of its ability to help 'address issues of educational equity and social exclusion, and open up democratic and educational opportunities ${ }^{\prime 62}$ and is generally regarded as to provide ease of access and flexibility, portability, improved student-teacher contact, and increased discussions with peers ${ }^{61}$. However, in the midst of convenience of online resources, it is warned that decreased access to teaching physicians, and dependence on the availability of technology, need to be addressed when using this modality ${ }^{15}$. The use of multimedia in medical teaching is also on the rise largely due to its 'special capacity of interactivity and it achieves its greatness through its interaction', as well as being able to provide greater retention of knowledge ${ }^{14}$, at the same time that electronic devices are playing a growing role in the academic lives of medical students, especially in Malaysia ${ }^{63}$. Virtual model, another increasingly popular method, is able to provide a 3-dimensional and dynamic view of the structures, and focus on user interaction with the model, thus enhancing understanding of the anatomical structures and physiological mechanisms, and providing an ideal platform for hands-on procedures. es and physiological mechanisms, and providing an ideal platform for hands-on procedures.

It has been noted that a mode of learning's acceptability is likely to influence its effectiveness ${ }^{61}$. This can be seen from our reviews as well as, among the 15 papers that favoured TEL in terms of student satisfaction, all apart from one demonstrated a significant improvement in knowledge or skill. Concurrently, all 3 papers favouring satisfaction with TL found no significant improvement in knowledge or skill, as well as 2 out of 3 paper showing a mixed, or no significant difference in student satisfaction. Moreover, it is crucial to note that all 3 papers that favoured traditional learning, did leave room for TEL as a useful accompanying tool, as it has been described as non-inferior to $\mathrm{TL}^{41}$, a useful adjunct to traditional methods ${ }^{52}$, and have an important role in reinforcing learning and aiding revision ${ }^{48}$. Thus, for TEL interventions to work effectively, policymakers and educators should strive to understand and address specific factors and aspects of TEL that positively influences students' acceptability. It has been noted that student satisfaction does not depend on the TEL format alone, but rather in establishing a strong educator presence in online settings and building online learning communities that foster positive relations ${ }^{64}$. The overwhelming majority of published papers on TEL from developed nations highlights the urgent need for more studies in evaluating and comparing the effectiveness of TEL amongst developing countries for better adaptation to local needs and conditions. In all, while TEL shows promising results, it is fair to note that it will play a hand-in-hand role as traditional learning, as it been expressed by a number of papers, viewing it as a supplementary tool alongside rather than a replacement one $6,36,56$.

\section{CONCLUSION}

In all, the findings present blended learning in a positive and promising light in time particularly where systematic reviews on technology-enhanced learning in the field of undergraduate medical program have mixed results. Nevertheless, with the advent of technological advances and the fundamental increment of the familiarity, experience and skills of educators in incorporating technology in teaching, it is just a natural phenomenon that increasingly complex tools are developed to support and enhance teaching in the undergraduate medical field. From the review, the authors recognize that there is an urgent need for more TEL evaluation studies to detail the purpose of TEL interventions and the assessment and overall approaches adopted, the economic properties of the interventions, the specific design of learning materials and to describe how has technology enhanced and impacted the students' learning experience. 


\section{REFERENCES}

1. Graffam B. (2007). Active learning in medical education: Strategies for beginning implementation. Med Teach 29: 38 - 42.

2. Lujan HL, Dicarlo SE. (2006). How We Learn too much teaching, not enough learning: what is the solution? Adv Physiol Educ 30: 17 - 22.

3. Roopa S, Geetha Bagavad M, Rani A, Chacko T. (2013). What type of lectures students want? - A reaction evaluation of dental students. J Clin Diagnostic Res 7: 2244 2246.

4. Alebaikan R, Troudi S. (2010). Blended learning in Saudi universities: Challenges and perspectives. ALT-J Res Learn Technol 18: 49 - 59.

5. Bencheva N. (2010). Learning Styles and E-Learning Face-to-Face to the Traditional Learning. E-learning $63-67$.

6. Dhir SK, Verma D, Batta M, Mishra D. (2017). E-learning in medical education in India. Indian Pediatr 54 (10): 871 - 877.

7. Sagheb MM, Amini M, Saber M et al. (2017). Teaching Evidence-Based Medicine (EBM) to Undergraduate Medical Students through Flipped Classroom Approach. Shiraz E-Medical J In Press 0 - 5.

8. Bickerdike S, Whittle SR, Pickering JD. (2014). Do lecture audio-recordings support engagement and flexible learning? Med Educ 48: 522.

9. Joynes V, Fuller R. (2016). Legitimisation, personalisation and maturation: Using the experiences of a compulsory mobile curriculum to reconceptualise mobile learning. Med Teach 38: 621 - 627.

10. Lumsden CJ, Byrne-Davis LMT, Mooney JS, Sandars J. (2015). Using mobile devices for teaching and learning in clinical medicine. Arch Dis Child Educ Pract Ed 100: 244 - 251.

11. Fuller R, Joynes V. (2015). Should mobile learning be compulsory for preparing students for learning in the workplace? $\mathrm{Br} J$ Educ Technol 46: 153 - 158.

12. Raja Hussain RM. (2004). eLearning in Higher Education Institutions in Malaysia. E-Mentor 5: $1-6$.

13. Allen LK, Eagleson R, de Ribaupierre S. (2016). Evaluation of an online three-dimensional interactive resource for undergraduate neuroanatomy education. Anat Sci Educ 9: 431 - 439.

14. Pickering JD. (2017). Measuring learning gain: Comparing anatomy drawing screencasts and paper-based resources. Anat Sci Educ 10: 307 - 316.
15. Vaccani JP, Javidnia $H$, Humphrey-Murto S. (2016). The effectiveness of webcast compared to live lectures as a teaching tool in medical school. Med Teach 38: $59-63$.

16. Higgins J, Green S. (2011). Cochrane handbook for systematic reviews of interventions Version 5.1. 0. The Cochrane Collaboration. Confid intervals.

17. Methley AM, Campbell S, Chew-Graham C et al. (2014). PICO, PICOS and SPIDER: a comparison study of specificity and sensitivity in three search tools for qualitative systematic reviews. BMC Health Serv Res 14: 579.

18. Harden RM, Grant J, Buckley G, Hart IR. (1999). BEME Guide No. 1: Best evidence medical education. Med Teach 21 (6): 553 - 562.

19. Moher D, Liberati A, Tetzlaff J, Altman DG. (2009). Preferred Reporting Items for Systematic Reviews and Meta-Analyses: The PRISMA Statement. Ann Intern Med 151: 264.

20. Ackermann O, Siemann H, Schwarting T, Ruchholtz S. (2010). Effektives training der chirurgischen röntgenbefundung durch E-learning. Z Orthop Unfall 148: 348 - 352.

21. Amesse L. (2008). Evaluation of Computeraided Strategies for Teaching Medical Students Prenatal Ultrasound Diagnostic Skills. Med Educ Online 13: 13.

22. Armstrong P, Elliott T, Ronald J, Paterson B. (2009). Comparison of traditional and interactive teaching methods in a UK emergency department. Eur J Emerg Med 16: 327 - 329.

23. Davidson LK. (2011). A 3-year experience implementing blended TBL: Active instructional methods can shift student attitudes to learning. Med Teach 33:750 753.

24. Ferrer-Torregrosa J, Torralba J, Jimenez MA et al. (2015). ARBOOK: Development and Assessment of a Tool Based on Augmented Reality for Anatomy. J Sci Educ Technol 24: $119-124$.

25. Green MJ, Levi BH. (2011). Teaching advance care planning to medical students with a computer-based decision aid. J Cancer Educ 26: $82-91$.

26. Gunn T, Jones L, Bridge P et al. (2017). The use of virtual reality simulation to improve technical skill in the undergraduate medical imaging student. Interact Learn Environ 1 - 8.

27. Hauer KE, Chou CL, Souza KH et al. (2009). Impact of an in-person versus web-based practice standardized patient examination on student performance on a subsequent high-stakes standardized patient examination. Teach Learn Med 21: 284 - 290. 
28. Ilic D, Nordin R Bin, Glasziou P et al. (2015). $A$ randomised controlled trial of a blended learning education intervention for teaching evidence-based medicine. BMC Med Educ 15: 39.

29. Ingrassia $\mathrm{PL}$, Ragazzoni L, Tengattini $\mathrm{M}$ et al. (2014). Nationwide program of education for undergraduates in the field of disaster medicine: Development of a core curriculum centered on blended learning and simulation tools. Prehosp Disaster Med 29: $508-515$.

30. Javadian P, Shobeiri S. (2016). Comparison of dissection-based vs . internet-based pelvic anatomy education for 3 rd year medical students. J Educ Technol Heal Sci 3: 38 - 41.

31. Jenkins S, Goel R, Morrell DS. (2008). Computer-assisted instruction versus traditional lecture for medical student teaching of dermatology morphology: A randomized control trial. J Am Acad Dermatol 59: 255 - 259.

32. Kandasamy T, Fung K. (2009). Interactive Internet-based cases for undergraduate otolaryngology education. Otolaryngol Head Neck Surg 140: 398 - 402.

33. Kanthan R, Senger J-LLJJL. (2011). The impact of specially designed digital gamesbased learning in undergraduate pathology and medical education. Arch Pathol Lab Med 135: 135 - 142.

34. Kerfoot BP, Brotschi E. (2009). Online spaced education to teach urology to medical students: a multi-institutional randomized trial. Am J Surg 197: 89 - 95.

35. Kleinert $\mathrm{R}$, Heiermann $\mathrm{N}$, Plum PS et al. (2015). Web-based immersive virtual patient simulators: Positive effect on clinical reasoning in medical education. J Med Internet Res 17: 1 - 11.

36. Kong J, Li X, Wang $Y$ et al. (2009). Effect of digital problem-based learning cases on student learning outcomes in ophthalmology courses. Arch Ophthalmol 127: 1211 - 1214.

37. Langdorf $M$, Anderson $C$, Navarro $R$ et al. (2018). Comparing the Results of Written Testing for Advanced Cardiac Life Support Teaching Using Team-based Learning and the "Flipped Classroom" Strategy. CureusCom 10: 1 - 9 .

38. Lewis EC, Strike M, Doja A et al. (2011). Webbased software to assist in the localization of neuroanatomical lesions. Can J Neurol Sci 38: $251-255$.
39. Miller M. (2016). Use of computer-aided holographic models improves performance in a cadaver dissection-based course in gross anatomy. Clin Anat 29: 917 - 924.

40. Mione S, Valcke M, Cornelissen M. (2013). Evaluation of virtual microscopy in medical histology teaching. Anat Sci Educ 6: 307 315.

41. Montassier E, Hardouin JB, Segard J, et al. (2016). E-Learning versus lecturebased courses in ECG interpretation for undergraduate medical students: A randomized noninferiority study. Eur J Emerg Med 23: 108 - 113.

42. Ochoa JG, Wludyka P. (2008). Randomized Comparison Between Traditional and Traditional Plus Interactive Web-Based Methods for Teaching Seizure Disorders. Teach Learn Med 20: 114 - 117.

43. Palmer EJ, Devitt PG. (2008). Limitations of student-driven formative assessment in a clinical clerkship. A randomised controlled trial. BMC Med Educ 8: 29.

44. Raupach T, Muenscher C, Anders $S$ et al. (2009). Web-based collaborative training of clinical reasoning: A randomized trial. Med Teach 31: 431 - 437.

45. Raupach T, Münscher $C$, Pukrop $T$ et al. (2010). Significant increase in factual knowledge with web-assisted problembased learning as part of an undergraduate cardio-respiratory curriculum. Adv Health Sci Educ Theory Pract 15: 349 - 356.

46. Ricks C, Ratnapalan S, Jain S, Tait G. (2008). Evaluating computer-assisted learning for common pediatric emergency procedures. Pediatr Emerg Care 24: 284 - 286.

47. Saltarelli AJ, Roseth CJ, Saltarelli WA. (2014). Human cadavers Vs. multimedia simulation: A study of student learning in anatomy. Anat Sci Educ 7: 331 - 339.

48. Schreiber BE, Fukuta J, Gordon F. (2010). Live lecture versus video podcast in undergraduate medical education: A randomised controlled trial. BMC Med Educ 10: 1 - 6. https://doi.org/10.1186/14726920-10-68.

49. Smits PBA, De Graaf $L$, Radon $K$ et al. (2012). Case-based e-learning to improve the attitude of medical students towards occupational health, a randomised controlled trial. Occup Environ Med 69: 280 $-283$. 
50. Stolz D, Langewitz W, Meyer A et al. (2012). Enhanced didactic methods of smoking cessation training for medical students- $A$ randomized study. Nicotine Tob Res 14: 224 $-228$.

51. Subramanian A, Timberlake M, Mittakanti $\mathrm{H}$ et al. (2012). Novel educational approach for medical students: Improved retention rates using interactive medical software compared with traditional lecture-based format. J Surg Educ 69: 449 - 452.

52. Stirling A, Birt J. (2014). An enriched multimedia eBook application to facilitate learning of anatomy. Anat Sci Educ 7: 19- 27.

53. Tian Y, Xiao W, Li C et al. (2014). Virtual microscopy system at Chinese medical university: An assisted teaching platform for promoting active learning and problemsolving skills. BMC Med Educ 14: 1 - 8.

54. Thompson AR, Lowrie DJ. (2017). An evaluation of outcomes following the replacement of traditional histology laboratories with self-study modules. Anat Sci Educ 10: 276 - 285.

55. Truncali A, Lee JD, Ark TK et al. (2011). Teaching physicians to address unhealthy alcohol use: A randomized controlled trial assessing the effect of a Web-based module on medical student performance. J Subst Abuse Treat 40: 203 - 213.

56. Webb AL ouis., Choi S. (2014). Interactive radiological anatomy eLearning solution for first year medical students: Development, integration, and impact on learning. Anat Sci Educ 7: $350-360$.
57. Vyas R, Albright S, Walker D et al. (2010). Clinical training at remote sites using mobile technology: An India-USA partnership. Distance Educ 31: 211 - 226.

58. Yeung JC, Fung K, Wilson TD. (2012). Prospective evaluation of a web-based three-dimensional cranial nerve simulation. J Otolaryngol - Head Neck Surg 41: 426 436.

59. Rui Z, Lian-Rui X, Rong-Zheng $Y$ et al. (2017). Friend or Foe? Flipped Classroom for Undergraduate Electrocardiogram Learning: A Randomized Controlled Study. BMC Med Educ 17: 1 - 9.

60. Rowe M, Frantz J, Bozalek V. (2012). The role of blended learning in the clinical education of healthcare students: A systematic review. Med Teach 34 (4): 216 - 221.

61. Al-Shorbaji N, Atun R, Car J et al. (2015). A systematic review informing a radical transformation of health workforce development. 156.

62. Majumder A, D'Souza U, Rahman S. (2005). Trends in medical education: challenges and directions for need-based reforms of medical training in South-East Asia. Indian J Med Sci 58: 369 - 380.

63. Rahman NIA, Aziz AA, Zulkifli Z et al. (2015). Perceptions of students in different phases of medical education of the educational environment: Universiti Sultan Zainal Abidin. Adv Med Educ Pract 6: 211 - 222.

64. Nortvig AMM, Petersen AK, Balle SH, Hattesen Balle S. (2018). A literature review of the factors influencing e-learning and blended learning in relation to learning outcome, student satisfaction and engagement. Electron J e-Learning 16: 46 - 55. 
